\title{
PROBABILISTIC ASSESSMENT OF PRESSURISED THERMAL SHOCKS
}

Vladislav Pistora, Miroslav Posta, Dana Lauerova

Division of Integrity and Technical Engineering, Nuclear Research Institute Rez, Czech Republic

E-mail of corresponding author: pis@ujv.cz

\section{ABSTRACT}

Rector pressure vessel (RPV) is a key component of all PWR and VVER nuclear power plants (NPPs). Assuring its integrity is therefore of high importance. Due to high neutron fluence the RPV material is embrittled during NPP operation. The embrittled RPV may undergo severe loading during potential events of the type of pressurised thermal shock (PTS), possibly occurring in the NPP. The resistance of RPV against fast fracture has to be proven by comprehensive analyses. In most countries (with exception of the USA), proving RPV integrity is based on the deterministic PTS assessment. In the USA, the "screening criteria" for maximum allowable embrittlement of RPV material, which form part of the USA regulations, are based on the probabilistic PTS assessment. In other countries, probabilistic PTS assessment is performed only at research level or as supplementary to the deterministic PTS assessment for individual RPVs.

In this paper, description of complete probabilistic PTS assessment for a VVER 1000 RPV is presented, in particular, both the methodology and the results are attached. The methodology corresponds to the Unified Procedure for Lifetime Assessment of Components and Piping in WWER NPPs, "VERLIFE", Version 2008. The main parameters entering the analysis, which are treated as statistical distributions, are as follows:

- initial value of material reference temperature $\mathrm{T}_{0}$,

- reference temperature shift $\Delta \mathrm{T}_{0}$ due to neutron fluence,

- neutron fluence,

- size, shape, position and density of cracks in the RPV wall,

- fracture toughness of RPV material (Master Curve concept is used).

The first step of the analysis consists in selection of sequences potentially leading to PTS, their grouping, establishing their frequencies, and selecting of representative scenarios within all groups. Modified PSA model is used for this purpose. The second step consists in thermal hydraulic analyses of the representative scenarios, with the goal to prepare input data for the structural analyses (pressure and temperature variations in the reactor downcomer). The third step consists in performing structural analyses, covering (deterministic) temperature and stress fields calculations for all representative scenarios, and finally, probabilistic fracture mechanics analyses. The results of the third step analyses are conditional probabilities of initiation of fast fracture for all selected representative scenarios. Combining them with frequencies of all groups, the final value of (unconditional) frequency of initiation of fast fracture of the RPV is established.

In this paper, examples of both input data and the results are presented.

\section{INTRODUCTION}

The assessment of RPV integrity from the point of view of its resistance against fast fracture is based on comparison of stress intensity factor $K_{I}$ calculated for assessed point(s) of crack front of the assessed crack (either real or postulated) with its allowable value (fracture toughness) $K_{I C}$ for selected PTS regimes. As most of input data are of stochastic nature, two main approaches can be used:

1) deterministic approach, where conservative values for the input data are used and a single PTS assessment for those data is performed. Result of this assessment is prediction whether either "RPV fails" or "RPV does not fail".

2) probabilistic approach, where some input data are treated as statistical distributions. Usually Monte Carlo simulations (i.e. many individual PTS assessments for sampled input data) are performed. Main stochastic parameters within the assessment are as follows: neutron fluence on the RPV wall, initial value of reference temperature and its shift due to irradiation, crack density, size, aspect ratio and crack position in the RPV. Result of this assessment is expressed in terms of frequency of fast fracture initiation or frequency of RPV failure.

In reality, each of these two approaches contains some features of the other one. E.g., in deterministic approach, the true "lower bound" fracture toughness curve cannot be used, but only a curve at some low level of 
probability of fast fracture initiation is used. On the other hand, within the probabilistic assessment many parameters are taken as deterministic ones (e.g. dimensions of RPV or its thermal and tensile properties).

Deterministic approach is used as "basic" in most countries. Only in the USA, the probabilistic approach was used as a basis for developing the screening criteria. Large project for re-establishing the screening criteria, as given by 10 CFR 50.61 [1], was organised by US NRC in recent years [2]. The overall approach used in [2] was taken as a basis for developing the methodology for probabilistic PTS assessment for VVER RPVs, as is given in VERLIFE Unified procedure [3]. Of course, some VVER specific features were incorporated (e.g. assessment fully based on Master Curve reference temperature $T_{0}$, or absence of axial welds in VVER RPVs).

In this paper, description of the methodology as given in [3] and examples of both input data and the results for one specific VVER 1000 RPV are presented.

\section{METHODOLOGY OF PROBABILISTIC PTS ASSESSMENT FOR VVER REACTOR PRESSURE VESSELS ACCORDING TO UNIFIED PROCEDURE VERLIFE, VER. 2008}

A simplified basic scheme of the probabilistic evaluation is seen in Fig. 1.

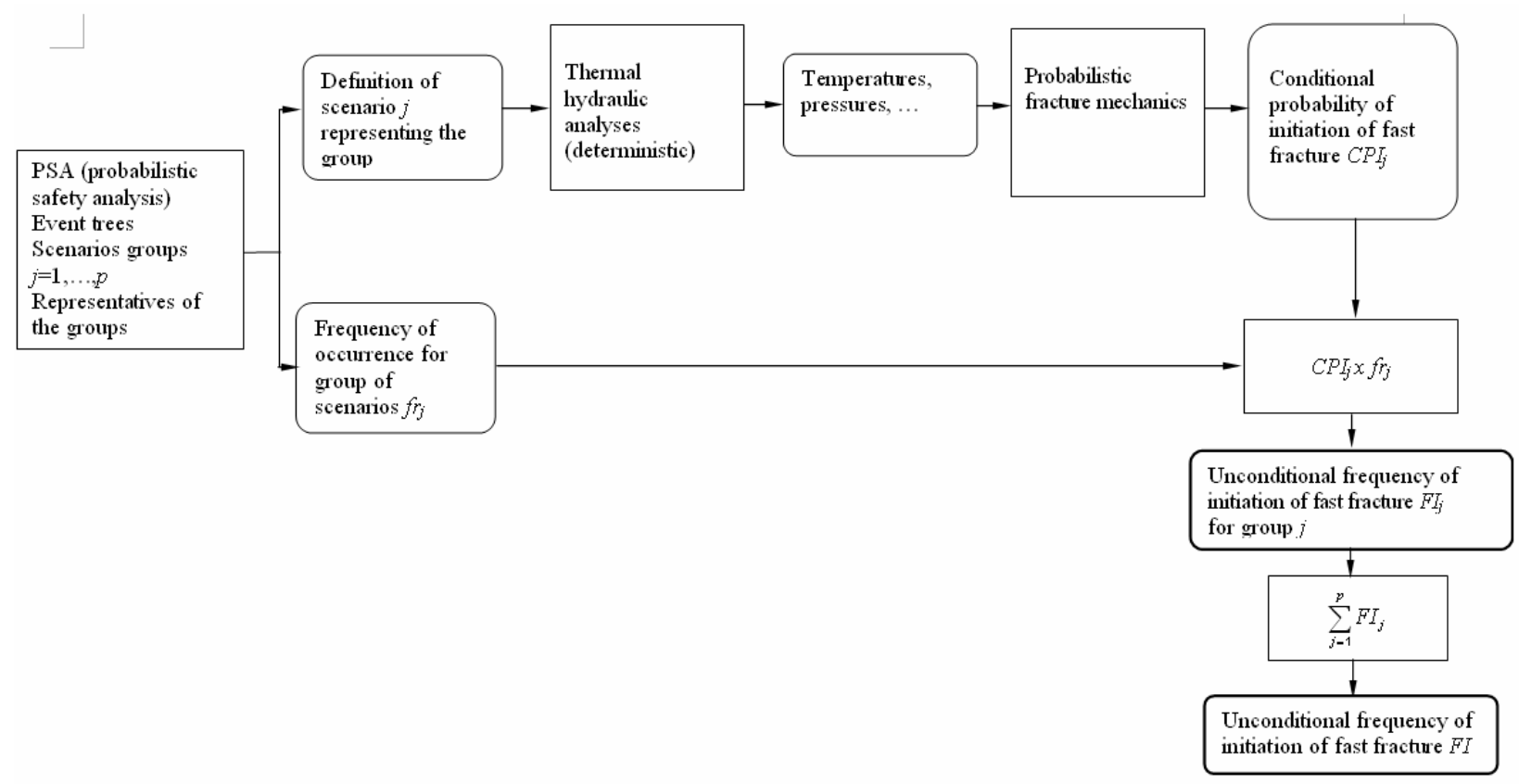

Fig. 1: Basic scheme of the probabilistic PTS evaluation

Within Probabilistic Safety Assessment (PSA), the event trees shall be developed for all initiating events potentially occurring in NPP that may lead to PTS. The identified scenarios have to be divided into different groups of similar variations of thermal hydraulic parameters, and from each group a representative has to be selected, having the worst impacts on RPV integrity from point of view of PTS. Using procedures of PSA, for each group the frequency of occurrence of individual PTS scenarios within the group will be determined, and (as a sum) the frequency of occurrence of the whole group, $f r_{j}$, will be established, including uncertainties (statistical distributions).

For all representatives selected, the conditional probabilities of fast fracture initiation, $C P I_{j}$, are determined. For less conservative evaluation, conditional probabilities of RPV failure, $C P F_{j}$, may be determined (i.e. conditional probabilities of crack propagation through RPV wall), provided that through-wall crack propagation after crack initiation as well as possible arrest of the crack are considered. In what follows, only the CPI approach is discussed. Under conditional probability of fast fracture initiation is understood probability of fast fracture initiation under condition that the particular scenario occurred.

Combining the frequency of $j$-th group $f r_{j}$ with corresponding conditional probability $C P I_{j}$, we obtain (unconditional) frequency of fast fracture initiation, for particular scenario group $F I_{j}$. Summing up the frequencies corresponding to all scenario groups, we obtain the final frequency of fast fracture initiation FI. Both scenario group 
frequencies $f r_{j}$ and conditional probabilities $C P I_{j}$, are statistical distributions, therefore the final frequency of fast fracture initiation is statistical distribution as well. For final evaluation its mean value should be used.

The main part of the assessment, establishing the conditional probabilities of fast fracture initiation, $C P I_{j}$, is described in Fig. 2. Basic part of the assessment are Monte Carlo simulations in which the values of individual parameters, which are of statistical nature, are randomly sampled from their statistical distributions - "random RPVs" with "random cracks" are generated. Parameters, which are treated as statistical distributions, and statistical assessments are highlighted by red colour in Fig. 2.

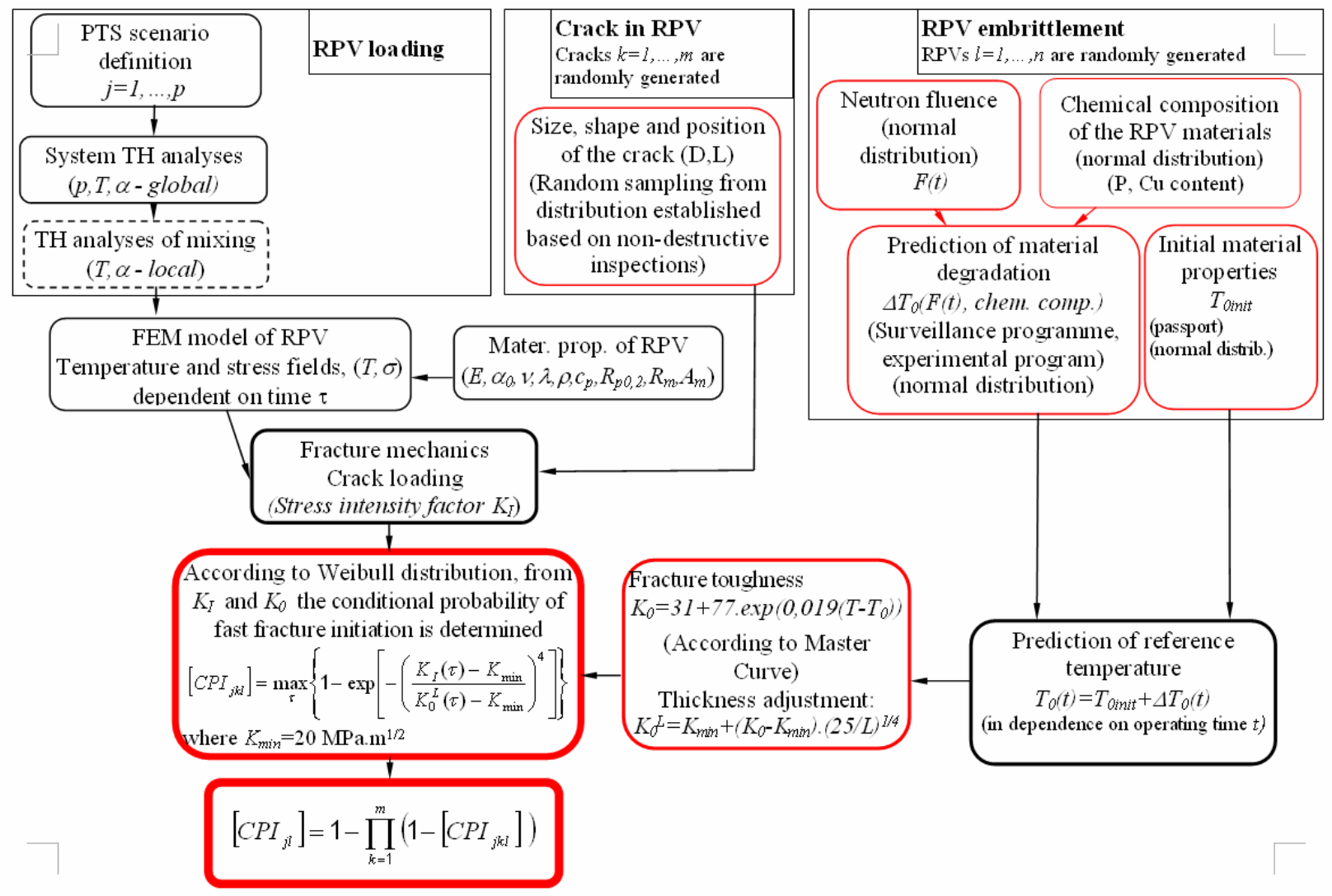

Fig. 2: Establishing the conditional probabilities of fast fracture initiation, $C P I_{j l}$

\subsection{Main Parameters Entering the Probabilistic PTS Assessment}

Main parameters entering the probabilistic PTS assessment, treated as statistical distributions (with indicated type of distribution, as proposed in [3]), are as follows:

\section{Randomly generated RPVs}

- $\quad$ Neutron fluence in dependency on time of RPV exploitation (Gaussian distribution)

- Chemical composition of RPV materials (namely $\mathrm{Cu}$ and P content, applied only in some cases) (Gaussian distribution)

- $\quad$ Initial value of Master Curve reference temperature $\boldsymbol{T}_{0}$ (Gaussian distribution)

- Shift of reference temperature $\boldsymbol{T}_{0}$ in dependency on neutron fluence (and chemical composition in some cases) (Gaussian distribution)

Types of cracks entering the evaluation

\section{- Underclad cracks}

Underclad cracks are generated in the base metal (not weld) of RPV wall and are positioned precisely under the cladding. They are assumed to be only axial, i.e. oriented in the direction perpendicular to the direction of cladding procedure. The underclad cracks may be generated as semi-elliptical or elliptical.

\section{- $\quad$ Embedded cracks}

Inner (embedded) cracks are those that lie fully inside the RPV wall and neither touch nor intersect cladding. All are assumed to have an elliptical shape. 


\section{a) Embedded cracks in the weld}

Embedded cracks in the weld are assumed to be circumferential only, due to the technology of welding.

b) Embedded cracks in base metal

$50 \%$ of embedded cracks in base metal are assumed to be circumferential and $50 \%$ of them are assumed to be axial.

\section{Statistical distribution of crack parameters Randomly generated cracks}

Random generating is performed based on statistical distributions of particular crack parameters that have to be determined from statistical treatment of data obtained for flaws detected in the evaluated RPV. These data may be obtained from manufacturing, pre-operational or in-service inspections. Statistical distributions of the crack parameters that are presented below are only recommended ones. Within statistical treatment of results of inspections also other distributions may be used, provided that it is demonstrated that they better describe the flaw data obtained.

- $\quad$ Number of cracks in unite volume of RPV material (density of cracks) (Poisson distribution)

- $\quad$ Crack depth treated as relative number of cracks of depth larger than $\mathrm{x}$ (exponential distribution)

- $\quad$ Difference of semiaxes of (semi)elliptical cracks (exponential distribution)

- Position of crack in RPV (uniform distribution along RPV wall thickness, relevant for embedded cracks only).

The relevant parameters of Poisson or exponential distributions are treated also as random values with Gamma distribution. The approach used in statistical treatment of cracks was taken from [4].

\section{Fracture toughness}

Statistical distribution of fracture toughness values is assumed to be the Weibull distribution, in accord with the Master Curve concept [5]. Median of fracture toughness values (for specimens of thickness $1 \mathrm{inch}=25,4 \mathrm{~mm}$ ) is assumed to be described through the following equation

$$
K_{J c}(\mathrm{med})=30+70 \exp \left[0.019\left(T-T_{0}\right)\right] .
$$

Master Curve concept is applied in such a manner that for a randomly generated reference temperature $T_{0}$ and actual temperature of material $T$ (dependent on time $\tau$ of the running PTS), value of Weibull distribution parameter $K_{0}$ is determined using the following equation (where a correction on crack length $L$ is considered):

$$
K_{0}(\tau)=20+\left[11+77 \cdot \exp \cdot\left(0,019 \cdot\left(T(\tau)-T_{0}\right)\right)\right] \cdot[25 / L]^{1 / 4} .
$$

\subsection{Determination of the Total Conditional Probability of Fast Fracture Initiation}

Based on time variation of $K_{I}$, the conditional probability of fast fracture initiation for time $\tau$ is determined (for crack $k$, vessel $l$ and scenario $j$ ):

$$
c p i_{j k l}(\tau)=1-\exp \left[-\left(\frac{K_{I}(\tau)-K_{\min }}{K_{0}(\tau)-K_{\min }}\right)^{4}\right],
$$

where $K_{\min }=20 \mathrm{MPa} \cdot \mathrm{m}^{1 / 2}$.

Conditional probability of fast fracture initiation over the entire PTS event is determined as its maximum over the time interval considered:

$$
C P I_{j k l}=\max _{\tau} c p i_{j k l}(\tau)
$$

Determination of mean value of conditional probability of fast fracture initiation for the selected scenario is schematically shown in Fig. 2.

Note: Fracture toughness values need not be randomly generated within this particular part of the evaluation (as are the other stochastic quantities entering the evaluation) and adequately, the probability of fracture need not be determined through the Monte Carlo method, but the CPI may be directly calculated according to Master Curve concept, using formula (3).

For determination of total conditional probability of fast fracture initiation $C P I_{j l}$ for $j$-th scenario and $l$-th vessel with $m$ randomly generated cracks the following relation is used: 


$$
C P I_{j l}=1-\prod_{k=1}^{m}\left(1-C P I_{j k l}\right),
$$

where $C P I_{j k l}$ is the conditional probability of initiation of the $k$-th crack for $j$-th scenario and $l$-th vessel.

The above-described procedure assesses the fast (brittle) fracture of RPV. For the "upper-shelf region", where ductile failure takes place, the analogous approach called "Master Curve for upper-shelf", as described in [6], and [7], was used.

\subsection{Determination of (Unconditional) Frequency of Occurrence of Fast Fracture Initiation}

Since the frequencies of occurrence for scenarios groups $\mathrm{fr}_{j}$ are stochastic quantities as well (their distributions are assumed to be known from the results of PSA), it is possible for each group of scenarios $j=1, \ldots, p$ to generate randomly the frequency of occurrence of group $f r_{j l}$ for each simulated RPV $l=1, \ldots, n$. as follows:

(Unconditional) frequency of occurrence of fast fracture for the $l$-th simulated vessel is then determined

$$
F I_{l}=\sum_{j=1}^{p} f r_{j l} \cdot C P I_{j l}
$$

In this manner we obtain a sample from the statistical distribution of frequency of occurrence of fast

fracture initiation. For the final evaluation its mean value $\overline{F I}$ is used.

The acceptance criterion for safe RPV operation according to [3] is

$$
\overline{F I}<1.10^{-6} / \text { reactoryear }
$$

\section{EXAMPLE: PROBABILISTIC ASSESSMENT OF PRESSURISED THERMAL SHOCKS FOR VVER 1000 REACTOR PRESSURE VESSEL}

\subsection{Selection of scenarios}

A specific PSA focussed on PTS was performed. The current "classical" PSA model, focussed on core damage, was used as a basis, but it had to be significantly modified, as events leading to PTS have in most cases opposite character than those leading to core damage. Finally, 54 groups of events were selected. The events were of the following types: LOCA, main steam line break, primary-to-secondary leak, and others. The highest frequency was found for the group of type "inadvertent opening of steam dump to atmosphere valve" with the mean frequency of occurrence $8,186 \cdot 10^{-6} /$ reactoryear. The groups with frequencies of occurrence less than $10^{-8} /$ reactoryear were screened out.

Within each group of events, a (conservative) representative scenario was selected, for which system thermal hydraulic analyses were performed using RELAP5 code. As the results of thermal hydraulic analyses, necessary for subsequent assessment, time variations of pressure, temperature and heat transfer coefficient in the reactor downcomer were obtained.

\subsection{Probabilistic fracture mechanics analyses}

For probabilistic fracture mechanics analyses the PROVER code developed in NRI Rez was used. This code is suitable for probabilistic assessment of PTS for VVER type RPVs. For each representative scenario, deterministic temperature and stress analyses were performed within the pre-processor of PROVER code using 1D finite elements.

The main part of the analysis consists in the probabilistic fracture mechanics analysis using Monte Carlo method. The sources of input data were as follows:

- $\quad$ Neutron fluences - results of neutron fluence calculations were used with variation in radial, axial and circumferential direction in the RPV beltline region. Values of fluences were calculated dependent on time of RPV exploitation. The standard deviation of fluence was taken from known uncertainty of fluence calculations as $20 \%$ of the mean value. 
- Initial value of Master Curve reference temperature $\boldsymbol{T}_{0}$ was taken from the initial state of the surveillance programme, separately for welds No. 3 and 4 (in beltline region) and for base material of ring between them. The standard deviations were taken from [3].

- $\quad$ Shift of reference temperature $\boldsymbol{T}_{\boldsymbol{0}}$ in dependency on neutron fluence was taken from [3] (for VVER 1000 $\mathrm{RPV}$ it is according to [3] independent of chemical composition) as

$$
\Delta T_{0}=A_{F} \cdot\left(F \cdot 10^{-22}\right)^{1 / 3},
$$

where $A_{F}=20^{\circ} \mathrm{C}$ for weld metal,

$A_{F}=23^{\circ} \mathrm{C}$ for base metal.

- $\quad$ Statistical distributions of cracks were taken from NDE results from in-service inspections.

\subsection{Results}

For the analyses, 200000 simulations of RPV were used. The typical resulting mean value of CPI for significant PTS representative scenarios is in the range $10^{-7}$ to $10^{-6} /$ reactoryear. The main results for 40 and 60 years of operation are presented in Table 1.

Table 1: Resulting frequencies of fast fracture initiation $(F I)$ in $1 /$ reactoryear.

\begin{tabular}{|c|c|c|c|c|c|}
\hline Years of operation & $50 \%$ quantile & Mean value & $90 \%$ quantile & $95 \%$ quantile & $99 \%$ quantile \\
\hline 40 & $3,145 \mathrm{e}-09$ & $\mathbf{9 , 9 2 1 e - 0 9}$ & $1,819 \mathrm{e}-08$ & $3,312 \mathrm{e}-08$ & $1,144 \mathrm{e}-07$ \\
\hline 60 & $7,466 \mathrm{e}-09$ & $\mathbf{1 , 9 3 5 e - 0 8}$ & $3,799 \mathrm{e}-08$ & $6,456 \mathrm{e}-08$ & $1,922 \mathrm{e}-07$ \\
\hline
\end{tabular}

It is seen, that the acceptance criterion Eq. (7) is fulfilled with large margin. Moreover, it is seen that there is a large difference between mean and median values - the distribution of $F I$ is very skewed. The dependency of $F I$ on mean fluence is presented in Fig. 3.

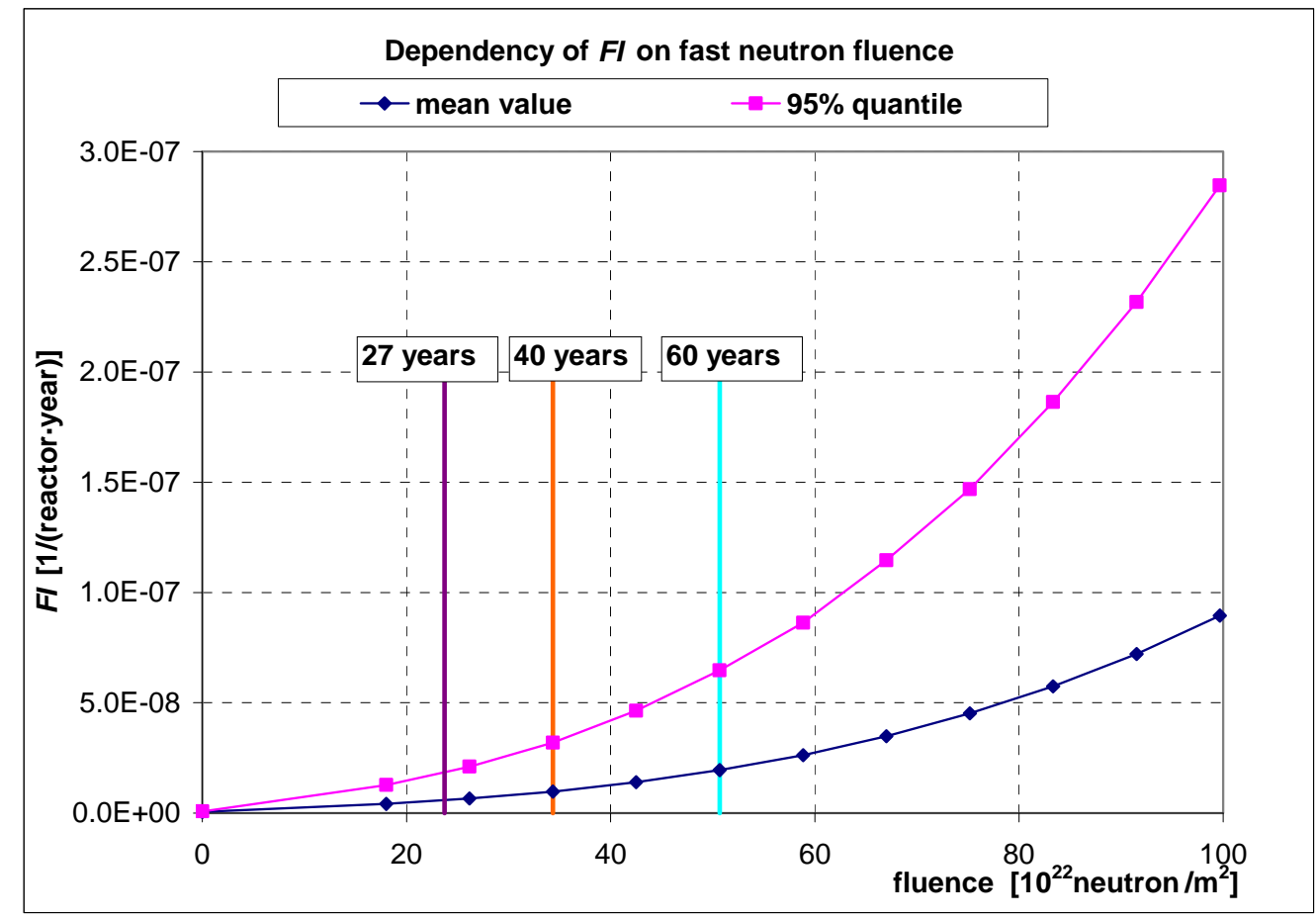

Fig. 3: Dependency of $F I$ on mean fluence.

Within the analyses, some supplementary interesting results were obtained. One of them is the contribution of individual RPV parts and the respective types of cracks (in beltline region) to the mean value of $F I$, see Table 2. The dependency of the contribution of individual types of cracks on mean fluence is presented in Fig. 4. 
Table 2: Relative contributions to mean $F I$ for individual regions and types of cracks for 60 years of operation

\begin{tabular}{|c|c|c|}
\hline Region & Crack type & Contribution to FI (\%) \\
\hline \multirow{3}{*}{ Lower cylindrical ring } & underclad & 10,5 \\
\cline { 2 - 3 } & axial & 0,5 \\
\cline { 2 - 3 } & circumferential & 0,1 \\
\hline Weld № 3 & circumferential & 16,1 \\
\hline \multirow{3}{*}{ Intermediate cylindrical ring } & underclad & 44,7 \\
\cline { 2 - 3 } & axial & 1,4 \\
\cline { 2 - 3 } & circumferential & 0,5 \\
\hline Weld №4 & circumferential & 20,4 \\
\hline \multirow{2}{*}{ Supporting ring } & underclad & 5,7 \\
\cline { 2 - 3 } & axial & 0,3 \\
\cline { 2 - 3 } & circumferential & 0,1 \\
\hline
\end{tabular}

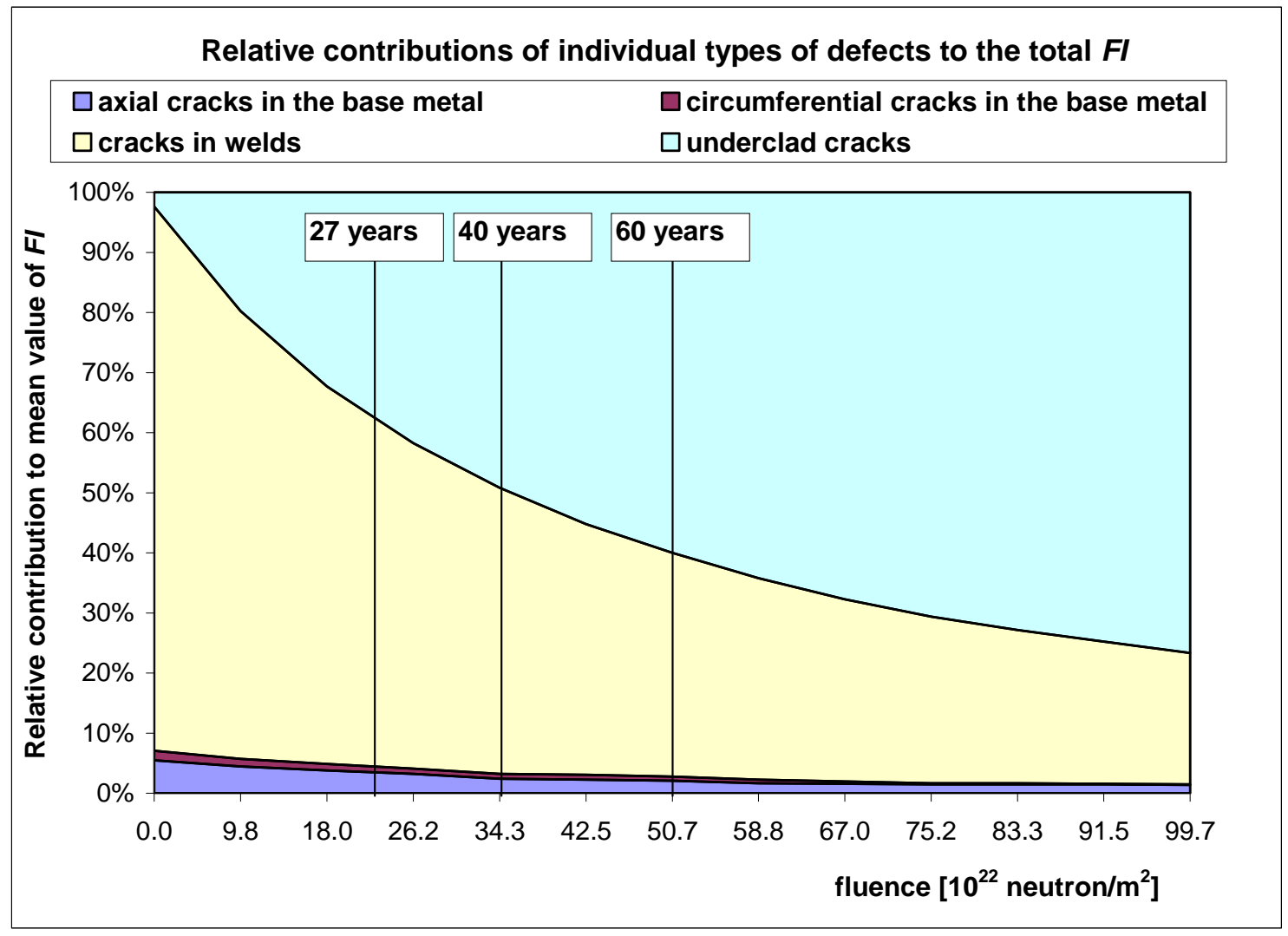

Fig. 4: Relative contributions of individual types of defects to total FI, in dependency on mean fluence.

It is seen that underclad cracks (due to their large number) and welds (due to their embrittlement) have dominant impact on the frequency of fast fracture initiation FI. The importance of underclad cracks is increasing with increasing RPV embrittlement.

Contributions of individual groups of scenarios and, as a result, of individual types of scenarios were assessed. In Fig. 5, the dependency of relative contributions of individual types of PTS regimes to the mean value of fast fracture initiation FI on fluence is presented. It is seen, that at the beginning of operation, when the RPV is not embrittled, flooding of RPV from outside is dominant, while during the operation, LOCA events become more significant. Within the group "OTHER", the events of type "inadvertent opening of pressurizer safety valve" play a main role. 


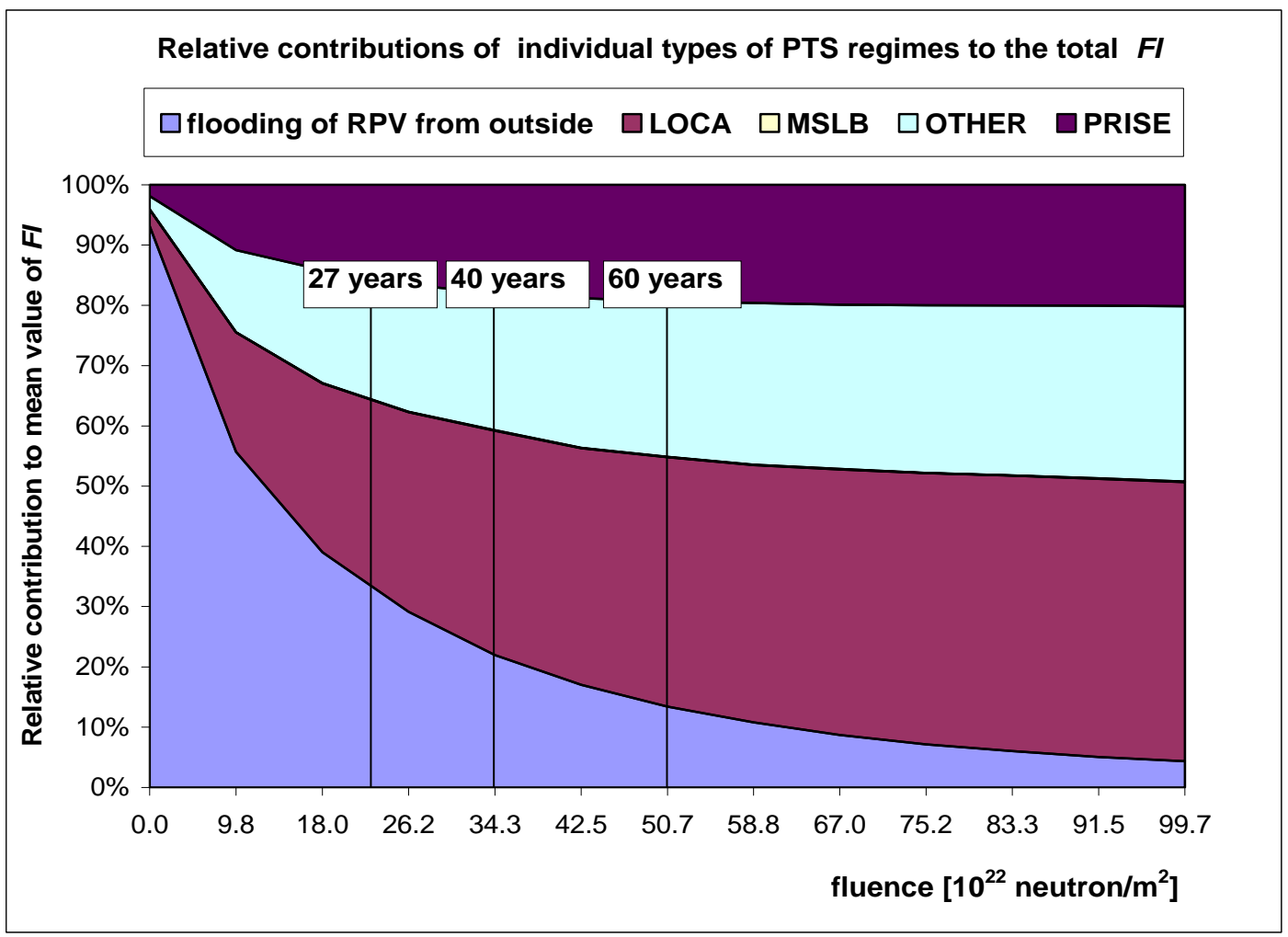

Fig. 5: Relative contributions of individual types of PTS regimes to mean value of fast fracture initiation FI, in dependency on fluence

\section{CONCLUSIONS}

Probabilistic assessment of pressurised thermal shocks was performed for VVER 1000 reactor pressure vessel according to Unified procedure VERLIFE [3]. The resulting mean value of fast fracture initiation frequency FI satisfies the acceptance criterion Eq. (7) as prescribed by the Unified procedure, with large margin.

The probabilistic assessment is used for VVER RPVs only as a supplementary one. The basic evaluation of integrity and lifetime of VVER RPV from the point of view of fast fracture is based on deterministic assessment.

\section{REFERENCES}

[1] Code of Federal Regulations 10 CFR 50.61, "Fracture Toughness Requirements for Protection Against Pressurized Thermal Shock Events"

[2] Technical Basis for Revision Of the Pressurized Thermal Shock (PTS) Screening Limit in the PTS Rule (10 CFR 50.61), NUREG-1806, US NRC

[3] Unified Procedure for Lifetime Assessment of Components and Piping in VVER NPPs, VERLIFE, ver. 2008 (prepared within the frame of COVERS project of the 6th Framework Programme of the EU)

[4] Simonen, F.A., Schuster, G.J., Doctor, S.R., Heasler, P.G.: A Generalized Procedure for Generating FlawRelated Inputs for the FAVOR-Code, NUREG/CR-6817, PNNL-14268, March 2003

[5] Standard Test Method for Determination of Reference Temperature, $T_{0}$, for Ferritic Steels in the Transition Range, ASTM E 1921-05

[6] EricksonKirk M.T., EricksonKirk M. A.: The relationship between the transition and upper-shelf fracture toughness of ferritic steels, Fatigue Fract Engng Mater Struct 29, p. 672 - 684.

[7] EricksonKirk M.T., EricksonKirk M. A., Roe C., Zhang X.J.: Use of Large Databases to Identify Trends in the Behavior of Ferritic Steels, Proceedings of the ASME 2009 Pressure Vessels and Piping Division Conference PVP 2009, July 26-30, 2009, Prague, Czech Republic. 